\title{
Overcoming Traditional Challenges in Nano-scale X-ray Characterization Using Independent Component Analysis
}

David Rossouw ${ }^{1}$, Pierre Burdet ${ }^{1}$, Francisco de la Peña ${ }^{1}$, Caterina Ducati ${ }^{1}$, Benjamin R. Knappett ${ }^{2}$, Andrew E. H. Wheatley ${ }^{2}$, Paul A. Midgley ${ }^{1}$.

1. Department of Materials Science and Metallurgy, University of Cambridge, 27 Charles Babbage Road, Cambridge, CB3 0FS, UK.

2. Department of Chemistry, University of Cambridge, Lensfield Road, Cambridge, CB2 1EW, UK.

Nano-heterostructures are inherently challenging to characterize due to the presence of spatially and often spectrally overlapping signals when using energy dispersive X-ray (EDX) spectroscopy or electron energy loss spectroscopy (EELS) techniques. In addition, inherently low signal yields from such small volumes and electron beam damage often limits signal quality. New image and spectral processing routes are needed to address these issues [1].

Here, we use a machine learning technique, called independent component analysis (ICA) [2], to unmix EDX signals originating from core-shell nano-magnetic particles. The ICA method, executed in HyperSpy [3], blindly decomposes the mixed signals into three components, which are found to accurately represent the isolated X-ray signals originating from the bi-metallic core, shell and supporting film (Fig. 1). The composition of the core is verified by and is in excellent agreement with the separate quantification of bare bi-metallic seed nanoparticles from the same synthesis. This validation provides the crucial evidence needed to justify the use of the technique in the analysis of individual phases in heterogeneous nano-scale volumes, without the need for their mechanical separation or separate analysis at each step in a multi-step synthesis procedure. The machine learning approach also efficiently handles noisy data, and can differentiate true spectral and spatial information from underlying noise, minimising the required sample beam dose [4]. 


\section{References:}

[1] G. Lucas, P. Burdet, M. Cantoni, and C. Hebert, Micron, 52 (2013), p. 49.

[2] C. Jutten and J. Herault, Signal Processing 24 (1991), p. 1.

[3] www.hyperspy.org

[4] D.R. acknowledges support from the Royal Society's Newton International Fellowship scheme. B.R.K. thanks the UK EPSRC for financial support (EP/J500380/1). F.d.1.P. and C.D. acknowledge funding from the ERC under grant no. 259619 PHOTO EM. P.A.M and P.B. acknowledges financial support from the European Research Council under the European Union's Seventh Framework Programme (FP7/2007-2013) / ERC grant agreement 291522-3DIMAGE. P.A.M. also acknowledges financial support from the European Union's Seventh Framework Programme of the European Commission: ESTEEM2, contract number 312483.

(a)

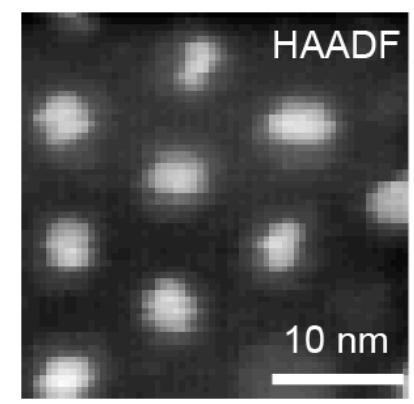

(b)

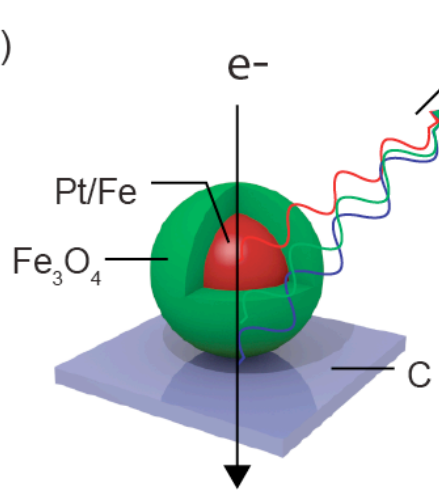

(c)

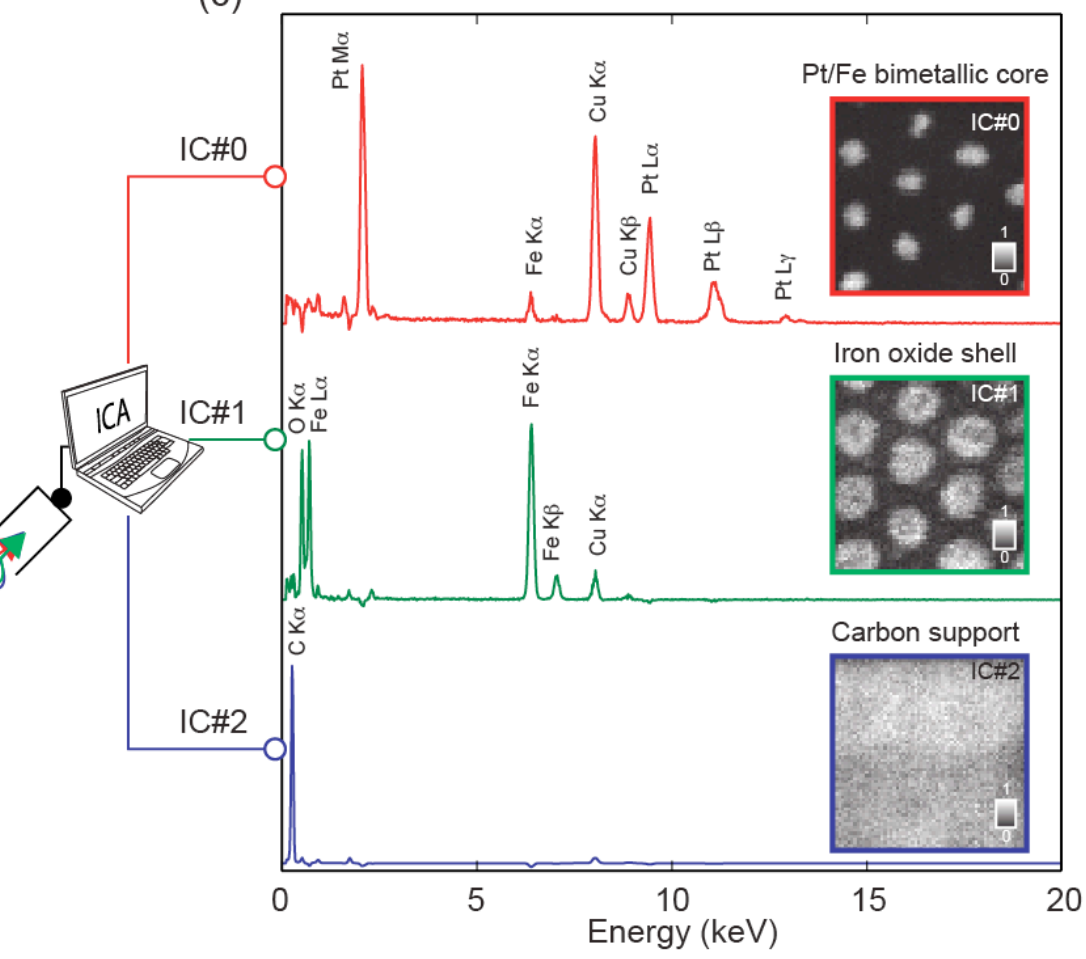

Figure 1. (a) An EDX spectrum image obtained from a cluster of core-shell nanoparticles. (b) The nanoparticles are comprised of a bi-metallic $\mathrm{Pt} / \mathrm{Fe}$ core surrounded by an iron oxide shell on a carbon support. (c) ICA decomposes the mixed EDX signals into components representing the core (IC\#0), shell (IC\#1) and support (IC\#2). 